\title{
Facet Joint Signal Change on MRI at Levels of Acute/Subacute Lumbar Compression Fractures
}

\author{
V.T. Lehman, C.P. Wood, C.H. Hunt, R.E. Carter, J.B. Allred, F.E. Diehn, J.M. Morris, J.T. Wald, and K.R. Thielen
}

\begin{abstract}
BACKGROUND AND PURPOSE: The prevalence of facet joint signal change in acute/subacute lumbar vertebral body compression fractures is unknown. We hypothesized that facet joint signal change on MR imaging is more common in facet joints associated with an acute/subacute lumbar compression fracture than those associated with normal vertebral bodies or ones that have a chronic compression fracture.
\end{abstract}

MATERIALS AND METHODS: Three neuroradiologists and a neuroradiology fellow retrospectively graded facet joint inflammatory change on MR imaging in 900 facet joints in 75 patients with at least 1 painful osteoporotic lumbar compression fracture. Facet joint signal change was assessed on T2-weighted images with chemical fat-saturation, STIR images, and/or gadolinium-enhanced T1-weighted images with chemical fat-saturation. Each facet joint from the T12/L1 to L5/S1 level was assessed individually. An overall facet joint signal-change score, which is a composite measure of the grade of signal change for all 4 facet joints associated with a given lumbar vertebral level, was devised, and statistical significance was assessed via Wilcoxon rank sum tests.

RESULTS: The overall facet joint signal-change scores were significantly higher at vertebral body levels affected by an acute/subacute compression fracture compared with control levels, which were associated with either normal bodies or chronic compression fractures.

CONCLUSIONS: Our findings suggest an association between facet joint signal change on MR imaging and acute/subacute lumbar vertebral body compression fractures.

ABBREVIATIONS: STIR $=$ short tau inversion recovery

$\mathrm{T}$ he exact source of pain from osteoporotic vertebral compression fractures and the mechanism of pain relief with vertebral augmentation remain unknown. There is recent evidence that pain associated with vertebral compression fractures can arise from the posterior elements, presumably from increased strain on facet joints. ${ }^{1,2}$ Microscopically, facet joint capsules are richly imbued with receptors sensitive to stretch or inflammation. ${ }^{3}$

While most literature indicates that chronic morphologic changes of facet joints are not predictive of facet joint pain, ${ }^{4}$ there is evidence that facet joint inflammation manifest as bone scan activity, facet joint hyperintensity on fat-suppressed T2-weighted MR imaging, or facet joint enhancement on gadolinium-enhanced MR imaging is predictive. ${ }^{5,6} \mathrm{MR}$ imaging evidence of facet

Received August 7, 2012; accepted after revision October 3.

From the Division of Neuroradiology (V.T.L., C.P.W., C.H.H., F.E.D., J.M.M., J.T.W., K.R.T.), Department of Radiology, Mayo Clinic College of Graduate Medical Education, Rochester, Minnesota; and Division of Biomedical Statistics and Informatics (R.E.C., J.B.A.), Mayo Clinic Department of Health Sciences Research, Rochester, Minnesota. Please address correspondence to Vance T. Lehman, MD, Department of Radiology, 200 First St SW, Rochester, MN 55905; e-mail: Lehman.vance@mayo.edu

http://dx.doi.org/10.3174/ajnr.A3449 joint signal change, defined as T2 hyperintensity or enhancement on fat-suppressed images, adjacent to compression fractures has not been characterized to date, to our knowledge. We hypothesized that evidence of facet joint signal change on MR imaging is more common in facet joints associated with an acute/subacute lumbar compression fracture than in facet joints adjacent to vertebral bodies that are either normal or have a chronic compression fracture.

\section{MATERIALS AND METHODS Patient Selection}

Approval of this Health Insurance Portability and Accountability Act-compliant study was obtained from the institutional review board. We searched the institutional vertebral augmentation data base for patients who underwent neuroradiologic evaluation for potential vertebral augmentation for painful lumbar osteoporotic compression fractures from May 2008 through June 2011. Fractures that occurred with absent or only minor trauma without evidence of pathologic vertebral body lesions were considered osteoporotic, even if a formal bone mineral attenuation was not available. This classification is consistent with criteria for a clinical 
diagnosis of osteoporosis, which can be made with either an osteoporotic fracture or with bone mineral attenuation testing. ${ }^{7}$ To be included, a patient had to be deemed an appropriate candidate for vertebral augmentation after a formal consult with an experienced neuroradiologist, had to have documented pain and tenderness at the level of vertebral augmentation, and then had to undergo the vertebral augmentation procedure in at least 1 lumbar level. These inclusion requirements were instituted to ensure that the selected patients indeed had a clinical pain syndrome that corresponded to the site of a compression fracture and that the patient group was one in which the potential findings of this study could most likely apply in clinical practice. An MR imaging including fat-suppressed T2-weighted images and/or fat-saturated gadolinium-enhanced T1-weighted images covering all facet joints from T12/L1 through L5/S1 at the time of fracture evaluation was also required. For patients who had undergone vertebral augmentation procedures on multiple days, only the most recent vertebral augmentation evaluation and/or procedure and the corresponding closest preceding MR images were analyzed to avoid redundancy.

\section{Exclusion Criteria}

Patients with traumatic nonosteoporotic vertebral body compression fractures or with pathologic fractures, such as those due to metastatic disease or multiple myeloma, were excluded. Patients with lumbar spine surgery within 6 months of the MR imaging examination were excluded to prevent confounding findings due to postoperative change. All patients with metallic hardware in the lumbar spine were excluded to avoid the effects of artifacts.

\section{Imaging}

At our institution, patients routinely underwent MR imaging examinations before evaluation for potential vertebral augmentation. Our vertebroplasty protocol included either sagittal fat-saturated T2-weighted images or sagittal STIR images, depending on the specific MR imaging scanner used and the radiologist's preference. In certain settings such as radiologist's preference, prior lumbar surgery, or evaluation for a possible vertebral body mass, gadolinium was administered. All MR imaging examinations were performed on a $1.5 \mathrm{~T}$ scanner.

\section{Image Analysis}

All images were reviewed at an electronic PACS workstation by 3 neuroradiologists (C.P.W., K.R.T., C.H.H.) and a neuroradiology fellow (V.T.L.) with 21 years, 16 years, 3 years, and 1 year of experience, respectively, for consensus diagnosis. At the time of review, readers were blinded to the original radiology report and contents of the neuroradiology vertebroplasty consult. Consensus review was performed to ensure that the interpretation generated was representative of a typical group of neuroradiologists. All lumbar levels were evaluated in each patient.

Sagittal fat-suppressed T2-weighted and/or gadolinium-enhanced T1-weighted images were the primary ones used to assess vertebral body edema and facet joint signal change. However, these images were reviewed in conjunction with each patient's entire examination, which typically included sagittal T1-weighted images; sagittal T2-weighted images without fat-suppression; and
Table 1: Grading of facet joint inflammation using fat-saturated MRI signal characteristics ${ }^{a}$

\begin{tabular}{cl}
\hline Grade & \\
\hline 0 & No signal abnormality \\
1 & Signal abnormality limited to joint capsule \\
2 & Periarticular signal abnormality involving $<50 \%$ of the \\
& facet joint perimeter \\
3 & Periarticular signal abnormality involving $>50 \%$ of the \\
& facet joint perimeter \\
4 & Grade 3 with additional signal abnormality within the \\
& neural foramen, pedicle, ligamentum flavum, transverse \\
& process, or vertebral body
\end{tabular}

${ }^{a}$ Grading scale adapted from Czervionke and Fenton. ${ }^{6}$

${ }^{\mathrm{b}}$ Periarticular signal abnormality includes $\mathrm{T} 2$ hyperintensity or gadolinium enhancement on Tl-weighted images.

'Signal abnormality within the pedicle must be continuous with other perifacet signal abnormalities and cannot represent posterior extension of vertebral body edema.

any available axial T2-weighted images, axial T1-weighted images, or axial gadolinium-enhanced T1-weighted images.

The vertebral bodies were evaluated for the presence or absence of an acute/subacute compression fracture, indicated by T2 hyper/T1 hypointense edema signal or a discrete visible fracture line, and for a chronic compression fracture, indicated by height loss without edema signal or a fracture line. Vertebral body edema is not a precise measure of compression fracture age because the duration after an osteoporotic compression fracture is not known with certainty. It is thought to typically resolve within 1-3 months, though edema in vertebral bodies treated with vertebral augmentation can remain for $>12$ months in a relative minority of cases ${ }^{8}$ and can even progress. ${ }^{9}$ Because the precise date of compression fracture for each patient was not known, vertebral body edema was considered a reasonable indicator of acute/subacute status.

The degree of vertebral body height loss, whether acute/subacute or chronic, was rated on a $1-4$ scale: $1=1 \%-25 \%$ height loss, $2=26 \%-50 \%$ height loss, $3=51 \%-75 \%$ height loss, and $4=>75 \%$ height loss. Prior vertebral augmentation procedures were recorded as present or absent at each level. Edema within vertebral bodies that had undergone prior augmentation was not considered evidence of acute/subacute status because vertebral body edema can persist in successfully treated compression fractures. ${ }^{8,9}$ The lowest mobile lumbar-type vertebral body was designated L5 in every patient for consistency.

Facet joint signal change was defined as T2 hyperintensity on fat-suppressed T2-weighted images and/or enhancement on fatsuppressed T1-weighted images. Although these signal changes have been previously referred to as inflammation, ${ }^{6}$ the term "signal change" was used because the precise physiologic cause of this signal change is not known with certainty. Facet joint signal change was graded by using an established scale of grades $0-4$ based on increasing osseous or soft-tissue signal abnormality at the facet joint (Table 1). ${ }^{6}$ To be consistent with the scale as used by Czervionke and Fenton, ${ }^{6}$ we graded a simple facet joint effusion without any other change zero because an effusion alone was not considered by these authors to necessarily indicate active inflammation or synovitis (personal communication L.F. Czervionke). All facet joints associated with the lumbar vertebral bodies were assessed individually, from the T12/L1 facet joints through the L5/S1 facet joints.

AJNR Am J Neuroradiol 34:1468-73 Jul 2013 www.ajnr.org 
Facet Joint Signal Change Score Schema for $L 2$ vertebral body

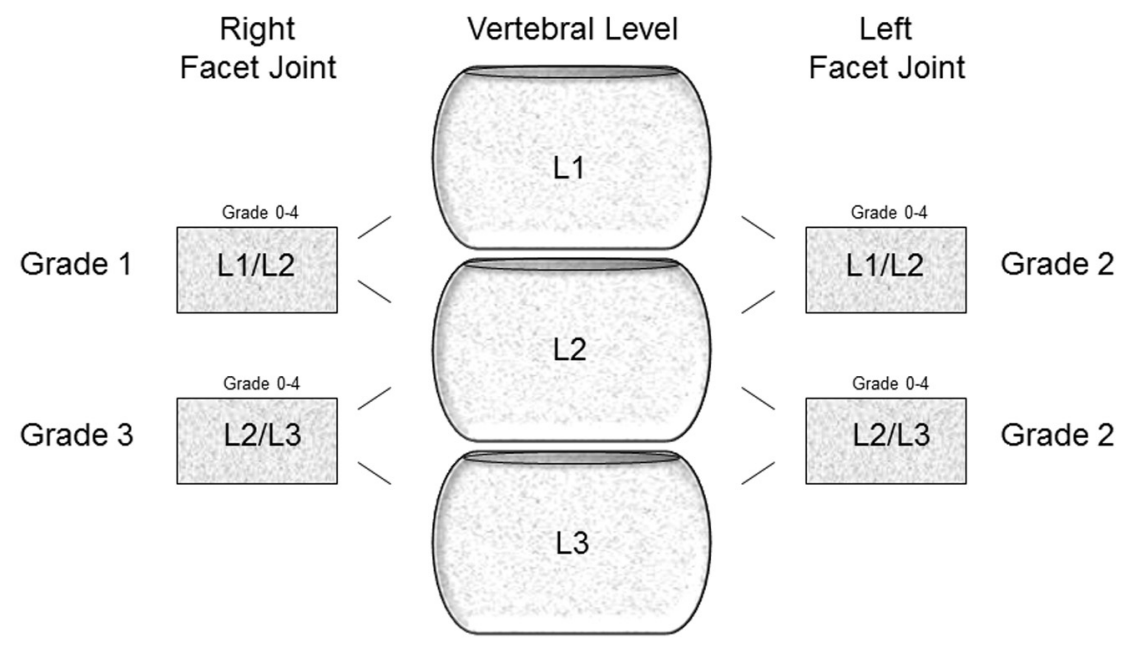

Signal Change Score of 8

FIG 1. For the L2 vertebral body, the facet joint signal-change score $(0-16)$ is the sum of the facet joint signal-change grades of each of the 4 associated facet joints ( $0-4)$. Anatomically, the $L 2$ vertebral body-associated facet joints are shared with the adjacent vertebral bodies. Therefore, the L2-associated facet joints could be subject to stress from compression fractures of the L2 or adjacent $\mathrm{L} 1$ and $\mathrm{L} 3$ vertebral bodies. For the $\mathrm{L} 2$ level, the above facet joint signal-change score for the L1/L2 and L2/L3 facet joints of patients with an acute/ subacute compression fracture of L1, L2, and/or L3 was compared with the facet joint signal-change score for the L1/L2 and L2/L3 facet joints of patients without an acute/ subacute compression fracture at L1, L2, or $\mathrm{L} 3$. This same method was then applied to the remaining lumbar vertebrae.

\section{Chart Review}

A neuroradiology consult was performed for every patient considered for vertebral augmentation at our institution. The dates of the consults and vertebral augmentation procedures and the levels of vertebral augmentation were recorded for each patient.

\section{Statistics}

The primary objective for statistical analysis was to determine whether the presence or degree of facet joint signal change was associated with the presence of an acute/subacute compression fracture. To assess this association, a facet joint signal-change score (Fig 1), which was the sum of the above signal-change grades $(0-4)$ for each of the 4 facet joints associated with a given vertebral body level, was calculated at each level, L1 through L5. This score represented an overall measure of facet joint signal change for an individual vertebral body level and ranged between 0 and 16. Each facet joint is associated with, and potentially affected by, a compression fracture of the vertebral body immediately above and immediately below it. To account for the potential effect of an acute/subacute fracture of an adjacent vertebral body on the shared facet joints, we considered a vertebral body level to be affected by an acute/subacute fracture if that vertebral body or either of the adjacent vertebral bodies had an acute/subacute fracture. For example, the L2 level (L1/L2 and L2/L3 facet joints) was considered to be affected by an acute/ subacute fracture if the L1, L2, and/or L3 vertebral body had an acute/subacute fracture.
Wilcoxon rank sum tests were then performed to determine whether these facet joint signal-change scores differed between vertebral body levels with an acute/subacute compression fracture versus those that were normal or had a chronic compression fracture. A levelby-level statistical analysis was performed because the biomechanical properties ${ }^{10}$ and baseline rate of facet joint signal change related to chronic $\operatorname{arthrosis}^{11}$ likely vary by level. Because there were few chronic compression fractures in our sample, a separate subgroup statistical analysis of this category was not performed. Finally, the degree of facet joint signal-change score was compared with the degree of height loss at levels with acute/subacute fractures by using the Spearman rank correlation.

Additionally, although the different levels were not combined for the formal statistical analysis, each of the 900 facet joints was also dichotomized into 2 groups: 1) facet joints associated with an acute/subacute compression fracture of either of the adjacent vertebral bodies, for example the L1 and L2 vertebral bodies for an L1/L2 facet joint; and 2) facet joints without an acute/subacute compression fracture of the adjacent vertebral bodies. Signal change was recorded as either present (any grade) or absent for each facet joint. Comparison of these 2 groups was performed to provide a more global view of the results, though this comparison did not account for potential differences in baseline inflammatory osteoarthrosis or biomechanical properties of the different levels.

\section{RESULTS}

\section{Patient Characteristics}

We identified 75 patients, 29 men and 46 women with a mean age of $74.7 \pm 12.1$ years, who met the inclusion criteria of the study. To reiterate, these patients underwent neuroradiology evaluation for potential lumbar vertebral augmentation for osteoporotic compression fractures between May 22, 2008, and June 30, 2011, had the requisite MR imaging available, and had at least 1 vertebroplasty performed.

We identified 8 additional patients who underwent vertebroplasty for osteoporotic compression fractures of the lumbar spine during this time, but they did not meet all the inclusion criteria. They were excluded for the following reasons: Three patients lacked fat-saturated T2-weighted images or fat-saturated gadolinium-enhanced T1-weighted images, 1 had recent spine surgery, 2 had incomplete coverage of the lumbar facet joints on MR imaging, 1 had metal lumbar spine hardware, and 1 had an upper lumbar compression fracture evaluated on thoracic MR imaging without dedicated lumbar imaging. 


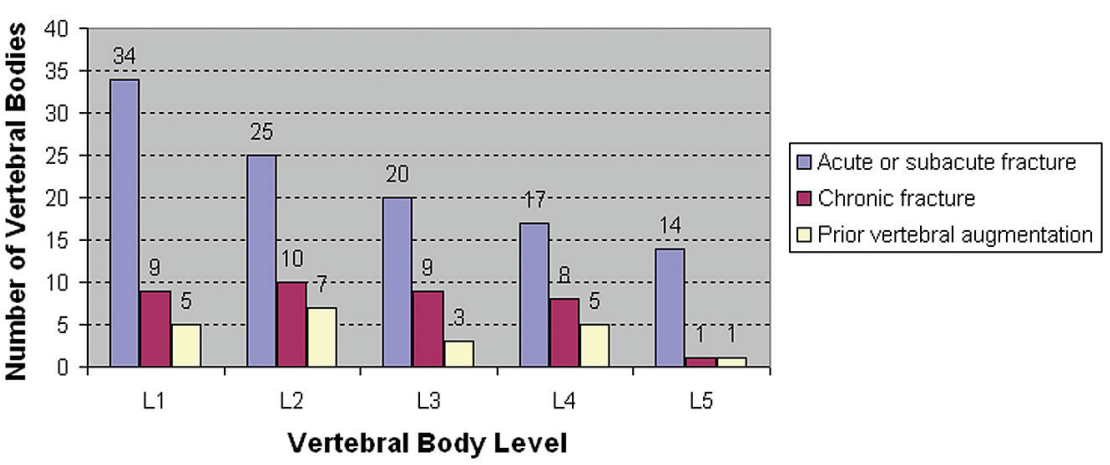

FIG 2. Level-by-level distribution of acute/subacute vertebral body compression fractures, chronic compression fractures, and prior vertebral augmentation.

Table 2: Facet joint signal-change scores of acute/subacute lumbar levels compared with other levels

\begin{tabular}{|c|c|c|c|c|c|}
\hline Facet Joint Signal-ChangeScore ${ }^{a}$ & & & & & \\
\hline Acute/sub & $2.2(3.3)$ & $2.6(3.3)$ & $3.7(3.7)$ & $4.6(5.2)$ & $4.8(5.3)$ \\
\hline Other (mean) (SD) ${ }^{b}$ & $0.0(0.0)$ & $0.3(0.9)$ & $1.0(2.3)$ & $1.2(2.5)$ & $0.4(0.9)$ \\
\hline$P$ value $^{c}$ & $<.01$ & .01 & $<.01$ & $<.01$ & $<.01$ \\
\hline
\end{tabular}

${ }^{a} A$ vertebral body was considered influenced by an acute or subacute fracture if either the body of interest or an adjacent vertebral body was fractured.

b "Other" is used to denote the complement to this case, namely vertebral bodies with no fracture or nearby fracture. Vertebral bodies with a chronic compression fracture could be considered "other" if there were no adjacent acute/subacute fractures

${ }^{c}$ Wilcoxon rank sum test.
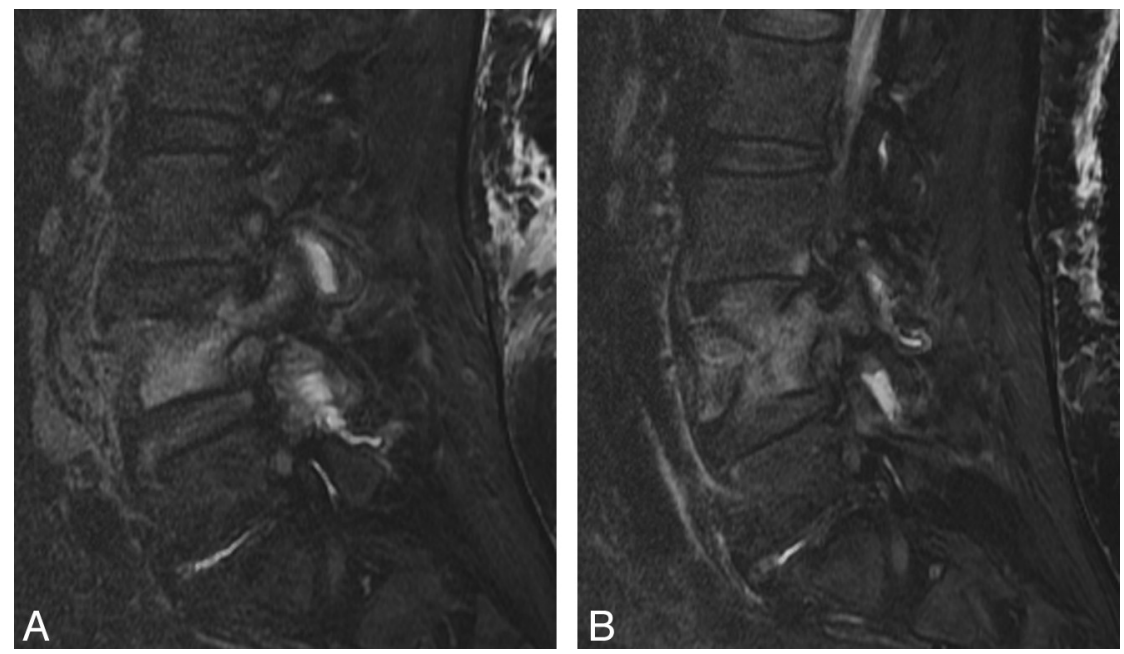

FIG 3. Facet joint inflammatory changes are more prevalent at levels of acute/subacute compression fracture than in levels without such fracture. A 66-year-old woman presented with an acute/subacute L4 compression fracture. Sagittal fat-saturated T2-weighted images demonstrate facet edema of the left $(A)$ and right $(B) L 3 / L 4$ and $L 4 / 5$ facet joints. There was no facet joint inflammatory change associated with the remaining lumbar facet joints (images not shown).

\section{Clinical and MR Imaging Evaluation}

The mean duration of the time between the MR imaging and clinical evaluation for potential vertebral augmentation was 5.9 days (range, 0-31 days), and the mean time between MR imaging and vertebral augmentation was 9.3 days (range, 0-43 days). Chemical fat-saturated T2 imaging was used in 66 patients, and STIR imaging was used in 9 patients. Ten patients additionally had fat-saturated T1 postgadolinium imaging. Nine hundred facet joints were independently assessed.

\section{Vertebral Body Compression Fracture Characteristics}

One hundred ten acute/subacute compression fractures were identified and evaluated in 75 patients. Single-level acute/subacute fractures were present in 53 patients $(71 \%)$. Multilevel fractures were present in $22 \mathrm{pa}-$ tients (29\%), including 2 fractures in 14 patients (19\%), 3 fractures in 4 patients (5\%), 4 fractures in 3 patients (4\%), and 5 fractures in 1 patient $(1 \%)$.

The level-by-level number of acute/subacute compression fractures, chronic compression fractures, vertebral bodies without compression fractures, and prior vertebral augmentation is outlined in Fig 2.

\section{Facet Joint Signal Change}

Table 2 depicts the facet joint signalchange scores for levels that were affected by an acute/subacute fracture and those that were not. There was a statistically significant increase in the overall facet joint signal-change score at all levels (L1-L5) in the presence of an acute/subacute compression fracture. An example of facet joint signal change associated with an acute/subacute compression fracture on MR imaging is depicted in Fig 3.

Facet joint signal-change scores were positively correlated with height loss at L1 $(P<.01)$, whereas an association was not found for levels L2-L5 (Table 3). There were, however, fewer instances of height loss for the inferior lumbar bodies. The distribution of degrees of vertebral body height loss across the 5 lumbar levels is outlined in Fig 4.

Overall, facet joint signal change (any grade) was present in $128 / 386$ (33\%) facet joints associated with an acute/subacute compression fracture compared with $40 / 514$ (8\%) facet joints that were not associated with an acute/ subacute fracture of either the vertebral body immediately superior or inferior to that facet joint. Of the facet joints with signal change associated with an acute/subacute compression fracture, $48 / 128$ (38\%) were immediately superior to the fracture level (eg, the L1/L2 facet joint relative to a compression fracture of the L2 vertebral body), 68/128 (53\%) were immediately inferior to the fracture level, and 12/128 (9\%) were sandwiched between adjacent compression fractures immediately above and below the facet joint.

\section{DISCUSSION}

The results of this study suggest an association between the prevalence and degree of facet joint signal change on MR imaging with 
Table 3: Facet joint signal-change score versus degree of height loss for patients with acute/subacute fractures

\begin{tabular}{lccccc}
\hline & L1 $(\boldsymbol{n}=\mathbf{7 5})$ & L2 $(\boldsymbol{n}=\mathbf{7 5})$ & $\mathbf{L 3}(\boldsymbol{n}=\mathbf{7 5})$ & $\mathbf{L 4}(\boldsymbol{n}=\mathbf{7 5})$ & $\mathbf{L} 5(\boldsymbol{n}=\mathbf{7 5})$ \\
\hline Spearman rank correlation $(\rho)$ & 0.41 & 0.15 & 0.02 & 0.22 & 0.14 \\
$P$ value & $<.01$ & .21 & .84 & .05 & .22 \\
\hline
\end{tabular}

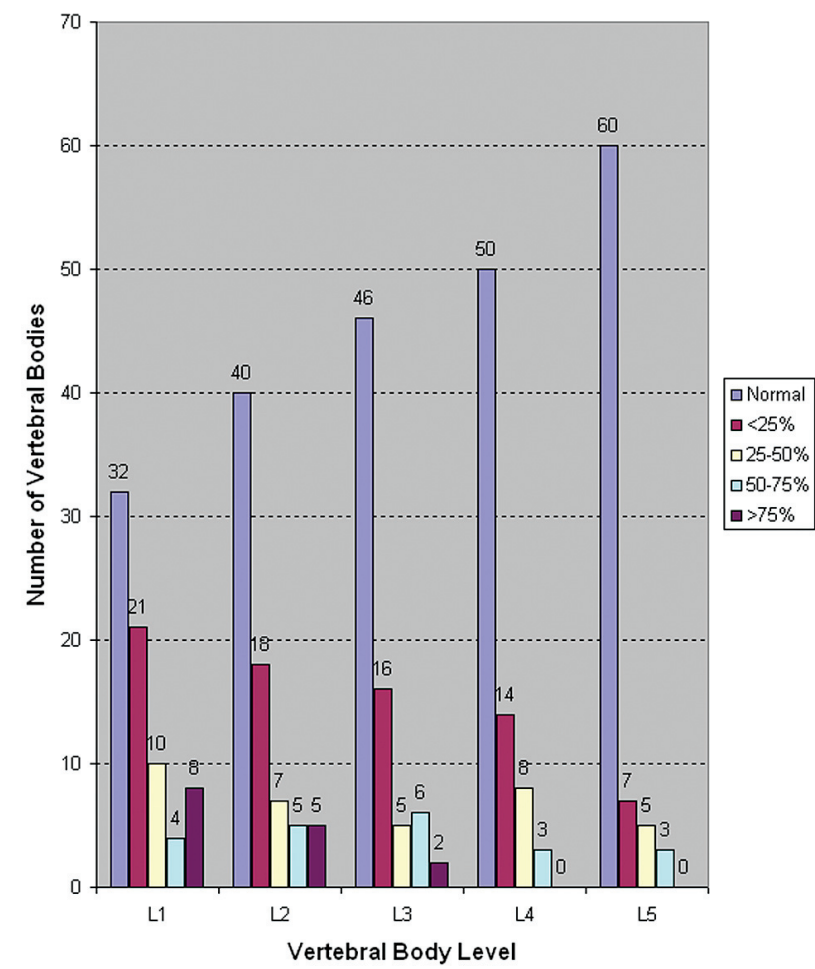

FIG 4. Level-by-level distribution of the degree of vertebral body height loss.

acute/subacute lumbar vertebral body compression fractures at all lumbar levels, compared with facet joints associated with vertebral bodies that were either normal or had a chronic compression fracture. This association was present for facet joints both immediately superior and immediately inferior to the acute/subacute fractures. We did not detect a correlation between facet joint signal change and the degree of compression fracture height loss at L2 through L5, though a positive correlation with the degree of compression at $\mathrm{L} 1$ was observed. These findings overall indicate that facet joint signal change can be associated with varying degrees of compression fracture height loss and is not restricted to only the most severe fractures.

Previous studies have demonstrated that facet joint signal change on MR imaging is associated with low back pain in patient populations without acute/subacute compression fractures. Czervionke and Fenton ${ }^{6}$ demonstrated a strong correlation between the side of axial low back pain and the presence of facet joint signal change on fat-saturated T2-weighted or gadolinium-enhanced T1-weighted MR images. Friedrich et $\mathrm{al}^{11}$ evaluated 145 patients with axial low back pain with STIR MR imaging and reported a $14 \%$ prevalence of facet joint T2 hyperintensity in this population. Most interesting, the overall prevalence of lumbar facet joint signal change in patients with acute/subacute compression fractures in our study (33\%) was higher than the overall prevalence reported in patients with low back pain in the study of Freidrich et al (14\%).

The physical cause of facet joint signal change has not been definitively established, to our knowledge. Previous work has referred to this as inflammation, ${ }^{6}$ which makes intuitive sense because facet joint inflammation is believed to be associated with pain resulting from a decreased threshold of nociceptor firing. ${ }^{12}$ This study does not determine the etiology of facet joint signal change in the setting of a compression fracture. However, one possibility is that biomechanical stress from the compression fracture incites facet joint inflammation and consequent signal change. This possibility is consistent with the findings of Friedrich et $\mathrm{al}^{11}$ of frequent facet joint $\mathrm{T} 2$ hyperintensity at the level of anterolisthesis or at the concave side of scoliosis, locations of probable biomechanical stress. ${ }^{11} \mathrm{~A}$ second but not mutually exclusive possibility is that the signal change is a reflection of direct injury to the facet joint and perifacet structures. Like inflammation, facet joint injury and stretch are thought to be potential causes of facet joint pain. ${ }^{12}$

The results of this study are relevant in the context of several recent studies that suggest that the posterior elements can be painful in the setting of a compression fracture. ${ }^{1,2,13}$ Bogduk et al ${ }^{1}$ have proposed and successfully tested a model to explain this concept, stipulating that facet joints must subluxate or tilt in the setting of a compression fracture. Wilson et al ${ }^{2}$ demonstrated a decreased need for vertebroplasty in patients first treated with facet joint injections. Kamalian et $\mathrm{al}^{13}$ showed that recurrent pain after vertebral augmentation can be treated with percutaneous facet and sacroiliac joint interventions. Other authors have suggested that pain relief in the control arms of the studies of vertebroplasty by Kallmes et al ${ }^{14}$ and Buchbinder et al ${ }^{15}$ might be explained by the treatment of posterior element pain with local anesthetic. $^{1,2}$ In contrast to our study, none of these studies discuss reviewing the facet joints for signal changes on cross-sectional imaging.

Given the findings of these studies, potential imaging markers of facet joint pain in the setting of vertebral body compression fractures could be useful for identification and potential treatment of a specific source of pain, separate from the acute/ subacute fracture of the vertebral body. Our study does not directly test whether specific facet joints with signal change associated with acute/subacute compression fractures were painful. Strictly speaking, the results of this study are an association of an imaging finding - facet signal change with vertebral compression fractures. The clinical utility of this imaging association is not definitively established with this study. However, our findings are important because future prospective studies could directly evaluate the likelihood of pain arising from facet joints, with signal change associated with an acute/ subacute compression fracture. Additional work could determine how to best manage and treat patients with acute/subacute compression fractures, such as targeting facet joints with signal change with steroid injections, medial branch blocks, and/or radio-frequency ablation rather than proceeding directly to vertebral augmentation. Facet joint signal change on 
fat-suppressed MR imaging is a reasonable marker to investigate because prior studies suggest that this finding is associated with facet joints that are painful and amenable to therapeutic intervention.

This study has several limitations. Because the facet joints are visualized on the same image sets as the compression fractures, we had no way to blind observers to the presence or acuity of compression fractures at the time of facet evaluation. Although control levels without compression fractures were examined in this study, unaffected control patients were not included. This occurred because fat-suppressed T2-weighted images and postcontrast fat-suppressed T1-weighted images for routine lumbar spine MR imaging (nonvertebroplasty evaluation) were inconsistently obtained at our institution until recently. Furthermore, this retrospective study did not permit inclusion of a fixed number of patients with injuries at each level of the lumbar spine. Therefore, the power to detect findings associated with facet joint signal change varied by level. This limitation is particularly relevant for the height-loss analysis because $<20 \%$ of patients had any degree of height loss at some vertebral body level. Because the number of chronic vertebral body compression fractures in this study was small and grouped with levels without fracture, a specific analysis of the association of facet joint signal change with chronic compression fractures was not possible.

\section{CONCLUSIONS}

Our findings suggest an association between facet joint signal changes on MR imaging and acute/subacute lumbar vertebral body compression fractures.

\section{ACKNOWLEDGMENTS}

We thank our study coordinator, Angela Majerus, for her assistance with data collection for this study and our departmental radiology research committee, which reviewed our study protocol and provided funding for statistical analysis.

Disclosures: Kent Thielen—UNRELATED: Royalties: Nevro Inc; Stock/Stock Options: Nevro Inc.

\section{REFERENCES}

1. Bogduk N, MacVicar J, Borowczyk J. The pain of vertebral body compression fractures can arise in the posterior elements. Pain Med 2010;11:1666-73

2. Wilson DJ, Owen S, Corkill RA. Facet injections as a means of reducing the need for vertebroplasty in insufficiency fractures of the spine. Eur Radiol 2011;21:1772-78

3. Cavanaugh JM, Ozaktay AC, Yamashita HT, et al. Lumbar facet pain: biomechanics, neuroanatomy, and neurophysiology. J Biomech 1996;29:1117-29

4. Cohen SP, Raja SN. Pathogenesis, diagnosis, and treatment of lumbar zygapophysial (facet) joint pain. Anesthesiology 2007;106:591-614

5. Pneumaticos SG, Chatziioannou SN, Hipp JA, et al. Low back pain: prediction of short-term outcome of facet injection with bone scintigraphy. Radiology 2006;238:693-98

6. Czervionke LF, Fenton DS. Fat-saturated MR imaging in the detection of painful facet arthropathy (facet synovitis) in the lumbar spine. Pain Med 2008;9:400-06

7. Qaseem A, Snow V, Shekelle P, et al. Screening for osteoporosis in men: a clinical practice guideline from the American College of Physicians. Ann Intern Med 2008;148:680-84

8. Voormolen MH, van Rooij WJ, van der Graf Y, et al. Bone marrow edema in osteoporotic vertebral compression fractures after percutaneous vertebroplasty and relation with clinical outcome. AJNR Am J Neuroradiol 2006;27:983-88

9. Dansie DM, Luetmer PH, Lane JI, et al. MRI findings after successful vertebroplasty. AJNR Am J Neuroradiol 2005;26:1595-600

10. Bogduk N. Clinical Anatomy of the Lumbar Spine and Sacrum. 5th ed. New York: Churchill Livingstone; 2012;29-37, 73-89

11. Friedrich KM, Nemec $S$, Peloschek $P$, et al. The prevalence of lumbar facet edema in patients with low back pain. Skelet Radiol 2007;36:755-60

12. Manchikanti L, Boswell MV, Singh V, et al. Comprehensive review of neurophysiologic basis and diagnostic interventions in managing chronic spinal pain. Pain Physician 2009;12:E71-120

13. Kamalian S, Bordia R, Ortiz AO. Post-vertebral augmentation back pain: evaluation and management. AJNR Am J Neuroradiol 2012;33:370-75

14. Kallmes DF, Comstock BA, Heagerty PJ, et al. A randomized trial of vertebroplasty for osteoporotic spinal fractures. $N$ Engl J Med 2009;361:569-79

15. Buchbinder R, Osborne RH, Ebeling PR, et al. A randomized trial of vertebroplasty for painful osteoporotic vertebral fractures. $N$ Engl J Med 2009;361:557-68 